\title{
DYNAMICS OF 2-DOF REGENERATIVE CHATTER DURING TURNING
}

\author{
NARESH K. CHANDIRAMANI ${ }^{1}$ and T. POTHALA \\ Dept. of Mechanical Engineering, Indian Intitute of Technology, Guwahati 781039, India
}

\begin{abstract}
A two-degree-of-freedom (2-DOF) model comprising nonlinear delay differential equations (DDEs) is analyzed for self-excited oscillations during orthogonal turning. The model includes multiple time delays, possibility of tool leaving cut, additional process damping (due to flank interference), ploughing force, and shear-angle/friction-angle variation. An algorithm to simulate tool dynamics and seek periodic solutions is developed based on two methods: (i) Direct numerical integration and (ii) Shooting for DDEs. The multipleregenerative and tool-leaving-cut effects are simulated via an equivalent 1-DOF system by introducing a time shift. The amplitude and minimum-period of limit cycles computed via these two methods compare well. Numerical studies involving the machining parameters are presented. The present plant model and dynamics could be useful for real time active control of tool chatter. ${ }^{1}$
\end{abstract}

\section{INTRODUCTION}

Self-excited vibrations that occur during machining are termed chatter. Chatter degrades surface finish and causes tool breakage. Passive control of chatter involves limiting the cutting parameters (like width and depth of cut), which in turn limits productivity. A pioneeing stability analysis of SDOF regenerative chatter was done by Tobias and Fishwick [1]. They considered the cutting force to be dependent on instantaneous chip thickness and feed velocity. Hanna and Tobias [2] modeled the structure with nonlinear stiffness and hysteretic damping, and the cutting force as a cubic polynomial in chip-thickness-variation (yielding nonlinear delay terms). Their analysis explained experimentally observed subcritical instability. Wu and Liu [3] considered a 2-DOF system with velocity-dependent friction and an empirical ploughing force. Besides experimental verification, their work showed that chip - rake friction caused chatter whereas interference between tool nose and work limited chatter. Berger et al. [4] used this model and obtained chaotic/aperiodic and limit-cycle dynamics during small and large amplitude chatter, respectively. Similar prechatter dynamics were experimentally confirmed by Johnson and Moon [5] who also presented a DDE model (SDOF). The 2-DOF model of Tlusty and Ismail [6] revealed that tool leaving cut improves stability. Nonlinearities due to tool leaving cut and due to interference between flank and machined wave (causing additional process damping) were considered by Jemielniak and Widota [7] and Tarng et al [8].

Ploughing force related damping, due to rounded tool nose and built up edge, was

\footnotetext{
${ }^{1}$ Corresponding author, naresh@iitg.ernet.in
} 


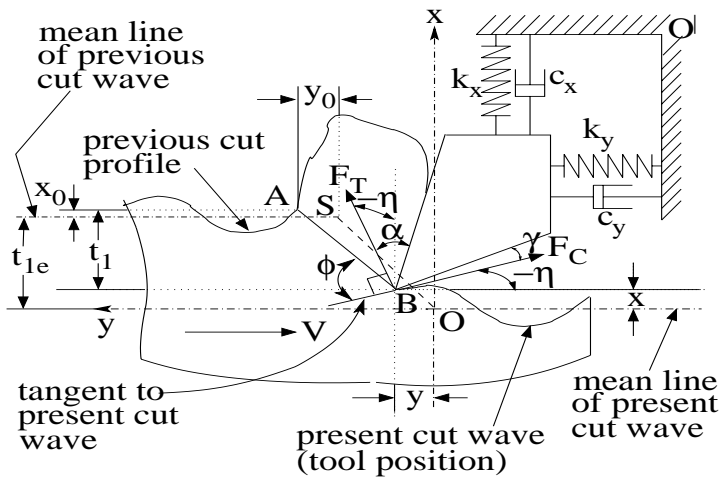

(a)

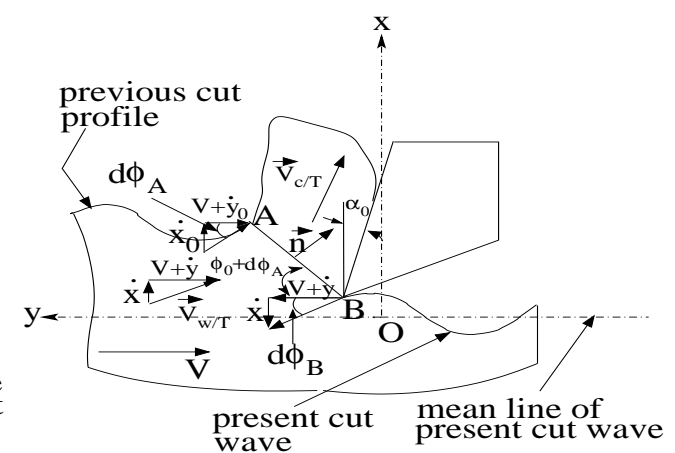

(b)

Figure 1: (a) Cutting tool system and coordinates; (b) Kinematics for shear angle [13] and $V_{C / T}[11]$

modeled by $\mathrm{Wu}$ [9] and Lee et al. [10] . Nosyreva and Molinari [11] considered velocity dependent friction and ploughing force. Their multiple scales analysis revealed saturation followed by reduction of amplitudes, in accord with experiments. Stepan [12] introduced an additional (short) regeneration time which distributes the force along tool-chip interface . The model predicted greater stability at high cutting speeds, as observed in experiments. Using a 2-DOF chatter model with multiple delays and shear angle variation, Lin and Weng [13] obtained chaotic chatter at certain widths of cut. In order to actively control turningtool chatter without reducing productivity, a plant model that contains all nonlinearities and effects aiding and countering chatter is required. Hence, in this paper a comprehensive, 2-DOF, non-linear model incorporating multiple regeneration, etc., is studied .

\section{EQUATIONS GOVERNING CHATTER}

Figure 1(a) shows the tool-work system. Origin $O$ of the $x-y$ system denotes the equilibrium (chatter free) position of the tool-tip. It moves (relative to work) along the $y$-axis at a velocity $V$ (cutting speed). The tool base $\left(O^{\prime}\right)$ and $O$ have identical motion. Point $B$ and line $B A$ denote the tool tip and the shear plane, respectively. During steady cutting, $B A$ coincides with $O S$. The displacement of $B$ and $A$, with respect to $O$ and $S$, respectively, are $(x, y)$ and $\left(x_{0}, y_{0}\right)$, respectively. Quantities $t_{1}, \phi, \alpha, \gamma$, and $\lambda$, denote uncut chip thickness, shear- ,rake-, clearance-, and friction- angles, respectively, and the corresponding ' $e$ '-subcripted quantity is the chatter-free value.

Referring to Fig. 1(a), the instantaneous uncut chip thickness is $t_{1}=t_{1_{e}}+x_{0}[t]-x[t]$. The shankwise chatter displacement (measured from the mean line) during the $n^{\text {th }}$-previous pass of the tool is $x_{n T}=x[t-n T]$. Here $T$ is the time for one revolution of work. During large chatter motions the tool could disengage from the work (i.e., $t_{1}<0$ ), causing the machining forces on the tool tip to vanish. Hence, the cut profile generated by the end of the previous pass is given as $x_{0}=\min \left[x_{1 T}, t_{1_{e}}+x_{2 T}, 2 t_{1_{e}}+x_{3 T}, \cdots\right]$. The multiple regenerative 
effect is due to this dependence of chip thickness on the chatter displacements of previous passes.

The shear angle varies due to tool vibration and waviness of previous-cut surface (Fig. 1(b)). Combining these effects yields [13],

$$
\phi=\phi_{e}+(\Delta \phi)_{A}+(\Delta \phi)_{B} ; \quad(\Delta \phi)_{A}=\tan ^{-1}\left[\dot{x}_{0} /\left(V+\dot{y}_{0}\right)\right], \quad(\Delta \phi)_{B}=\tan ^{-1}[-\dot{x} /(V+\dot{y})]
$$

Steady state cutting tests show that the chip-rake friction coefficient, $\mu(=\tan [\lambda])$, varies as $\mu=\mu_{0} \exp \left[-m_{\mu} V_{C / T}\right]$ [3]. The coefficients $\mu_{0}$ and $m_{\mu}$ are experimentally determined. The velocity of chip relative to tool $\left(V_{C / T}\right)$ is obtained using the continuity, across the shear plane, of the relative velocity [11]. This yields

$$
V_{C / T}=\frac{(V+\dot{y}) \sin \left[\phi_{e}+(\Delta \phi)_{A}\right]-\dot{x} \cos \left[\phi_{e}+(\Delta \phi)_{A}\right]}{\cos \left[\phi_{e}+(\Delta \phi)_{A}-\alpha_{e}\right]}
$$

The chatter-free damping coefficients $\left(c_{x}, c_{y}\right)$ represent structural and cutting process damping. Additional cutting process damping arises due to interference of the tool flank with the downward-inclined wavy machined surface $[7,8]$. Hence, the total damping coefficients are

$$
h_{x}=c_{x}\left(1-\mathcal{H}[-\dot{x}] \mu_{x} \eta / \gamma\right) ; \quad h_{y}=c_{y}\left(1-\mathcal{H}[-\dot{x}] \mu_{y} \eta / \gamma\right)
$$

Here $\mathcal{H}[\cdot]$ is the Heaviside function and $\eta$ is the angle (positive counterclockwise) between the $x$-axis and the normal to the wavy surface (Fig. 1(a)). Hence, one obtains $\eta=\tan ^{-1}[\dot{x} /(V+$ $\dot{y})$ ] and $\alpha=\alpha_{e}-\eta$. As is evident, the ratio $-\eta / \gamma$ increases[decreases] as the tool moves down a convex[concave] surface, and hence the additional damping increases[decreases], as expected, due to increasing[decreasing] interference between flank and machined wave.

Due to the (slight) roundededness of tool nose and the formation of built-up-edge (which increases the effective nose radius), a portion of the work material gets displaced (i.e., extruded) under the tool. Thus a ploughing force $\left(f_{x}, f_{y}\right)$ is exerted on the tool. Following [9], the ploughing force components are considered as $f_{x}=f_{s p} V_{d m}$ and $f_{y}=-\mu_{c} f_{x}$. Here $V_{d m}, f_{s p}$, and $\mu_{c}$, are the displaced volume of work, specific ploughing force, and friction coefficent between tool nose and displaced work (assumed constant), respectively. The volume of displaced work material is

$$
V_{d m}=w\left[\frac{V}{V \tan \gamma_{e}+\dot{x}}-\frac{V^{2}}{\left(V \tan \gamma_{e}+\dot{x}\right)^{2}} \frac{\tan \gamma_{e}}{2}\right] \psi^{2}
$$

Here $\psi$ is the depth of tool penetration (assumed constant), and $w$ is the width of cut (ie., chip width measured perpendicular to $x-y$ plane).

The cutting $\left(F_{C}\right)$ and thrust $\left(F_{T}\right)$ forces (Fig. 1(a)) are given by the Merchants Circle relations, i.e.,

$$
F_{C}=\frac{w t_{1} \tau_{s} \cos [\lambda-\alpha]}{\sin [\phi] \cos [\phi+\lambda-\alpha]} ; \quad F_{T}=\frac{w t_{1} \tau_{s} \sin [\lambda-\alpha]}{\sin [\phi] \cos [\phi+\lambda-\alpha]}
$$

where $\tau_{s}$ is the ultimate shear strength. Considering the cutting-, thrust-, ploughing-, and total damping- forces, the equations governing the chatter motions are written as (Fig. 1),

$$
\begin{aligned}
m \ddot{x}+h_{x} \dot{x}+k_{x} x & =\left(-F_{C} \sin [\eta]+F_{T} \cos [\eta]+f_{x}-\left(F_{T}+f_{x}\right)_{e}\right) \mathcal{H}\left[t_{1}\right] \\
m \ddot{y}+h_{y} \dot{y}+k_{y} y & =\left(-F_{C} \cos [\eta]-F_{T} \sin [\eta]+f_{y}-\left(-F_{C}+f_{y}\right)_{e}\right) \mathcal{H}\left[t_{1}\right]
\end{aligned}
$$



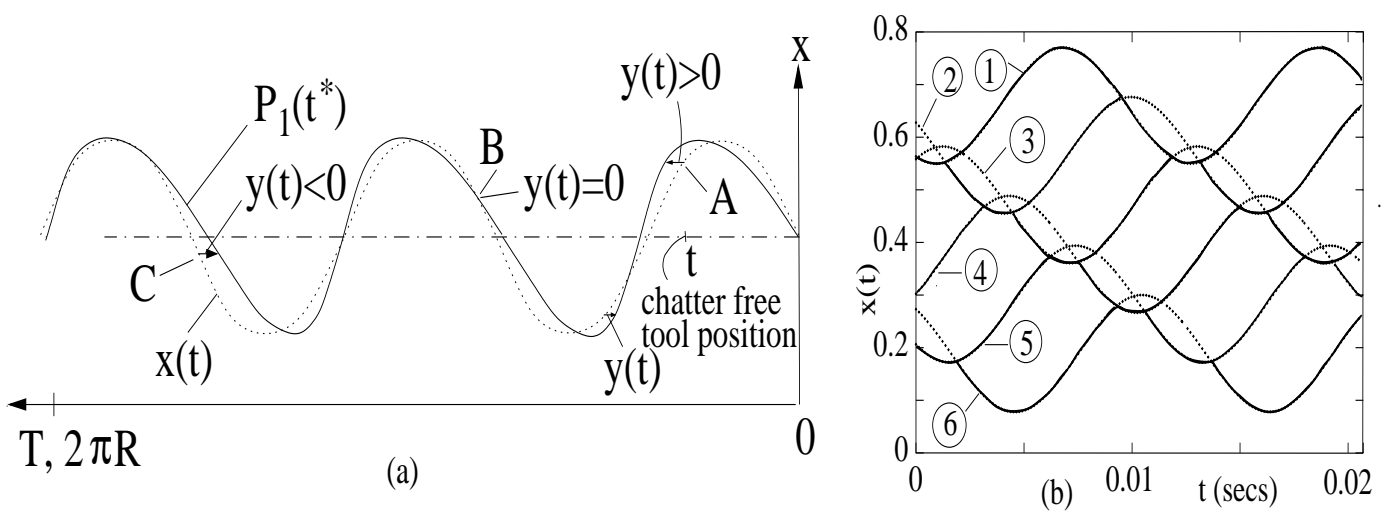

Figure 2: (a) Time shift; (b) Multiple Regeneration

where $(\cdot)_{e}$ denotes chatter-free evaluations (i.e., $x=\dot{x}=y=\dot{y}=0$ ). These are nonlinear delay differential equations (DDEs) as they contain the multiple regenerative effect (arising due to dependence of $F_{C}$ and $F_{T}$ on $t_{1}$, and characterized by multiple delay terms). The tool leaving cut effect appears via $\left.\mathcal{H}\left[t_{1}\right]\right)$.

\section{NUMERICAL SOLUTION}

The state vector is defined as $\left\{X_{1}, X_{2}, X_{3}, X_{4}\right\}^{T} \equiv\{x, \dot{x}, y, \dot{y}\}^{T}$. The resulting system of DDEs, i.e., $\dot{\mathbf{X}}[t]=\mathbf{F}[t, \mathbf{X}[t], \mathbf{X}[t-\tau]]$ subject to $\mathbf{X}[t]=\mathbf{\Phi}[t]=\mathbf{0}$ for $-\tau \leq t \leq 0$, is numericaly integated using the RK-4 method. The delay $\tau$ equals $n T$ (integer $n$ is the previous-pass number corresponding to the point $x_{0}$ on the previous cut-profile). Integration is performed over interval $[0, T]$ (i.e., one revolution of workpiece). Figure 2(a) shows the vector sum of $x$ and $y$ chatter displacements, i.e, the present displacement-profile $P_{1}$. The horizontal axis denotes time and, equivalently, the distance along the work circumference. Thus relations $x[t]=P_{1}\left[t^{*}\right], t^{*}=t+y / V$, hold. Thus, by using this time shift the effect of $y$-chatter is incorporated in the present-displacement profile, and consequently in the previous-cut profile. The instantaneous chip thickness is calculated using both these profiles, and then used to obtain machining forces. If $t_{1}>0\left[t_{1}<0\right]$ the tool is inside[outside] the cut and machining forces are non-zero[zero]. A step-size bisection is used to determine the instant when the tool leaves or enters the cut.

At the end of the integration interval, the previous cut-profile is updated (using interpolation) with that state - chosen out of the present-displacement and previous-cut profiles - that has mimimum $X_{1}\left[t^{*}\right]$. The updated cut-profile is used (with interpolation), during the next interval of integration, as the initial function $(\boldsymbol{\Phi}[t])$ and also to obtain $t_{1}$. The Poincarè surface $\left\{\left(X_{1}, X_{2}, X_{3}, X_{4}\right): X_{3}=0, X_{4}>0\right\}$ is defined, and relative differences in $X_{1}, X_{2}, X_{4}$ are computed over succesive surface intersections. If these differences remain small for the $i^{t h}$ and $(i+m)^{t h}$ intersection ( $m$ being the smallest positive integer) then convergence to a $m$-period limit cycle occurs and the period is the interval between these two intersections. A trivial fixed point is obtained if the intersections are always trivial. All 
integrations are done with unshifted time $t$ as the integration variable.

\section{Direct Integration Algorithm}

This entails forward time integration for a specified number of revolutions. The algorithm is:

[0] Initialize $\boldsymbol{\Phi}[t]$ (intial function) and Profile to zero. (Profile contains $t^{*}, X_{1}, X_{3}, X_{4}$, corresponding to previous cut-profile).

[1] Integration over $p^{\text {th }}$ pass $[0, T]$ : Integrate over step $\left[t_{I}, t_{O}\right]$; Perform time shift $t_{O}^{*} \leftarrow$ $t_{O}+y\left[t_{O}\right] / V$; Obtain present displacement-profile $\mathbf{P}\left[t_{O}^{*}\right] \leftarrow \mathbf{X}\left[t_{O}\right]$; Using interpolation access Profile at $t_{O}^{*}$ to extract $\mathbf{X}_{0}\left[t_{O}^{*}\right]$, i.e., the state vector corresponding to previous cut-profile; Calculate instantaneous chip thickness $t_{1} \leftarrow t_{1_{e}}+X_{1_{0}}\left[t_{O}^{*}\right]-P_{1}\left[t_{O}^{*}\right]$; If $t_{1}$ changes sign, i.e., tool leaves or enters the cut, use bisection; Tooldisp $\leftarrow\left(t_{O}^{*}, \mathbf{P}\left[t_{O}^{*}\right]\right)$.

[2] Updating Profile: Using interpolation Profile $\leftarrow\left(t_{O}^{*}, \min _{X_{1}}\right.$ (Profile, Tooldisp)).

[3] Check convergence to limit cycle or fixed point. Exit if converged or if number of passes exceeds prescribed limit, else continue at [1]. If converged to limit cycle then find amplitude and minimum period.

Figure 2(b) shows the multiple regenerative effect, similar to that reported in [6, 7]. Each continuous wave represents tool displacement-profile of the pass number indicated against it. Solid lines represent machined surface (cut profile) and dotted ones indicate tool disengagement. The displacement-profile for each pass is referenced to the mean-cut line (chatter-free) of that pass. Mean lines of succesive passes appear at a distance $t_{1_{e}}$ below. The tool leaves/enters the cut multiple times during a pass. It is observed that the displacement-profile of a pass intersects those of its subsequent two passes only, i.e., the delay is at most $2 T$. Hence, in order to implement the following shooting method $\boldsymbol{\Phi}[t]$ is assumed to be defined over $[-2 T, 0]$.

\section{Shooting Method for Periodic Solutions}

When using direct integration, converegence to a periodic state could be very slow for certain parameter ranges and initial conditions. Hence, a shooting method is considered. This iterative technique uses Newtons method to converge to a periodic solution, if one exist. Following Luzyanina et al. [14] one seeks an intial function (i.e., $\boldsymbol{\Phi}^{*}[t]$ defined over $[-2 T, 0]$ ) lying on the limit cycle, and the associated period $\mathcal{T}^{*}$. Let $\mathbf{X}_{\mathcal{T}}[\boldsymbol{\Phi}]$ denote the segment of the solution obtained over $[\mathcal{T}-2 T, \mathcal{T}]$ subject to initial function $\boldsymbol{\Phi}$. In order to impose the condition that the intial function being sought lies on the limit cycle, we consider the residue equation system $\mathbf{r}[\boldsymbol{\Phi}, \mathcal{T}] \triangleq \mathbf{X}_{\mathcal{T}}[\boldsymbol{\Phi}]-\mathbf{\Phi}=\mathbf{0}$. This system, being indeterminate, is augmented with a suitably chosen auxillary scalar equation $s[\boldsymbol{\Phi}, \mathcal{T}]=0$. An iterative solution of these equations would thus converge to $\left(\boldsymbol{\Phi}^{*}, \mathcal{T}^{*}\right)$. A first-order Taylor's expansion of these equations yields,

$$
\left[\begin{array}{cc}
\left(\partial \mathbf{X}_{\mathcal{T}} / \partial \boldsymbol{\Phi}\right)^{(i)}-\mathbf{I} & \left(\partial \mathbf{X}_{\mathcal{T}} / \partial \mathcal{T}\right)^{(i)} \\
(\partial s / \partial \boldsymbol{\Phi})^{(i)} & (\partial s / \partial \mathcal{T})^{(i)}
\end{array}\right]\left\{\begin{array}{c}
\Delta \boldsymbol{\Phi}^{(i+1)} \\
\Delta \mathcal{T}^{(i+1)}
\end{array}\right\}=-\left\{\begin{array}{c}
\mathbf{r}\left[\boldsymbol{\Phi}^{(i)}, \mathcal{T}^{(i)}\right] \\
s\left[\boldsymbol{\Phi}^{(i)}, \mathcal{T}^{(i)}\right]
\end{array}\right]
$$

Introduce notation $\left(\partial \mathbf{X}_{\mathcal{T}} / \partial \boldsymbol{\Phi}\right)^{(i)} \equiv \mathbf{S}^{(i)},\left(\partial \mathbf{X}_{\mathcal{T}} / \partial \mathcal{T}\right)^{(i)} \equiv \mathbf{g}^{(i)},(\partial s / \partial \mathbf{\Phi})^{(i)} \equiv \mathbf{c}^{(i)}$, and $(\partial s / \partial \mathcal{T})^{(i)} \equiv d^{(i)}$. The superscript ${ }^{(i)}$ in the coefficient matrix and in the driving vector (right hand side) denote evaluations at the solution $\left(\boldsymbol{\Phi}^{(i)}, \mathcal{T}^{(i)}\right)$ obtained after the $i^{\text {th }}$ 
iteration. An initial guess $\left(\boldsymbol{\Phi}^{(0)}, \mathcal{T}^{(0)}\right)$ of $\left(\boldsymbol{\Phi}^{*}, \mathcal{T}^{*}\right)$ is chosen. The algorithm for the $(i+1)^{\text {th }}$ iteration $(i=0,1,2, \ldots)$ is: (i) Integrate the DDE's over $\left[0, \mathcal{T}^{(i)}\right]$ subject to initial function $\boldsymbol{\Phi}^{(i)}$; (ii) Evaluate the coefficient matrix (using finite differences) and the driving vector, and solve Eq.(8) to obtain corrections $\left(\Delta \boldsymbol{\Phi}^{(i+1)}, \Delta \mathcal{T}^{(i+1)}\right)$; (iii) Use $\Delta \boldsymbol{\Phi}^{(i+1)}$ and $\Delta \mathcal{T}^{(i+1)}$ to test convergence. If convergence test passes then $\left(\boldsymbol{\Phi}^{*}, \mathcal{T}^{*}\right)$ (converged solution) has been obtained, else iterate the solution, i.e., $\boldsymbol{\Phi}^{(i+1)}=\boldsymbol{\Phi}^{(i)}+\Delta \boldsymbol{\Phi}^{(i)}, \mathcal{T}^{(i+1)}=\mathcal{T}^{(i)}+\Delta \mathcal{T}^{(i)}$.

Implementation entails discretization of the $n$-dimensional initial function vector at $(N+1)$ equally spaced time stations within $[-2 T, 0]$, i.e., $\boldsymbol{\Phi}^{(i)}=\left\{\phi_{11}^{(i)}, \ldots, \phi_{1 k}^{(i)}, \ldots, \phi_{1(N+1)}^{(i)}, \ldots\right.$, $\left.\phi_{j 1}^{(i)}, \ldots, \phi_{j k}^{(i)}, \ldots, \phi_{j(N+1)}^{(i)}, \ldots, \phi_{n(N+1)}^{(i)}\right\}^{T}$. Step (i) is performed using the discretized initial function (with interpolation between stations, if required). Using interpolation on the integrated solution vector, the discretized solution vector $\mathbf{X}_{\mathcal{T}}^{(i)}=\left\{x_{\mathcal{T}}(i), \ldots, x_{\mathcal{T}}^{(i)}{ }_{n(N+1)}^{(i)}\right\}^{T}$ is obtained at $(N+1)$ stations within $\left[\mathcal{T}^{(i)}-2 T, \mathcal{T}^{(i)}\right]$. The discretized driving vector is now readily obtained. Introduce the equivalent indexing $l \equiv(j-1)(N+1)+k$, e.g., $\phi_{l}^{(i)} \equiv \phi_{j k}^{(i)}$. The discretized $(n(N+1)) \times(n(N+1))$ matrix $\mathbf{S}^{(i)}$ is obtained via. finite differences. Using the initial function with $l^{\text {th }}$ element perturbed, i.e., $\tilde{\boldsymbol{\Phi}}^{(i)}=\left\{\phi_{1}^{(i)}, \ldots, \phi_{l}^{(i)}+\epsilon, \ldots, \phi_{(n N+n)}^{(i)}\right\}^{T}$, the perturbed solution vector $\tilde{\mathbf{X}}_{\mathcal{T}}^{(i)}=\left\{\tilde{x}_{\mathcal{T} 1}^{(i)}, \ldots, \tilde{x}_{\mathcal{T}(n N+n)}^{(i)}\right\}^{T}$ is obtained. Then $S_{m l}^{(i)}=\left(\tilde{x}_{\mathcal{T} m}^{(i)}-x_{\mathcal{T} m}^{(i)}\right) / \epsilon$, $m=1, \ldots, n N+n$. This procedure is done for $l=1, \ldots, n N+n$. The discretized $(n N+n)$-dimension vector $\mathbf{g}^{(i)}$ is obtained by perturbing $\tilde{\mathcal{T}}^{(i)}=\mathcal{T}^{(i)}+\epsilon$ and comput$\operatorname{ing} g_{l}^{(i)}=\left(x_{\tilde{\mathcal{T}} l}^{(i)}-x_{\mathcal{T} l}^{(i)}\right) / \epsilon$ using the unperturbed initial function $\mathbf{\Phi}^{(i)}$.

The discretized auxillary equation is specified as $s^{(i)} \triangleq \sum_{l=1}^{n N+n} F_{l}^{(i)}\left(\phi_{l}^{(i)}-x_{\mathcal{T}_{l}}{ }^{(i)}\right)$. Here $F_{l}^{(i)}$ represents the discretization of the time derivative of solution $\mathbf{x}_{\mathcal{T}}$ or, equivalently, the driving vector (RHS) in the state equations. The $(n N+n)$ discretized vector $\mathbf{c}^{(i)}$ is computed as $c_{l}^{(i)}=\left(\tilde{s}^{(i)}-s^{(i)}\right) / \epsilon$ where $\tilde{s}^{(i)}$ is based on $\mathcal{T}^{(i)}$ and $\tilde{\mathbf{\Phi}}^{(i)}$ with $l^{\text {th }}$ element perturbed. Further, $d^{(i)}=\left(\tilde{s}^{(i)}-s^{(i)}\right) / \epsilon$ where $\tilde{s}^{(i)}$ is based on $\tilde{\mathcal{T}}^{(i)}$ and $\boldsymbol{\Phi}^{(i)}$. Hence Eq. (8) represents a system of size $(n(N+1)+1)$. If $\left|\Delta \phi_{l}^{(i+1)} / \phi_{l}^{(i)}\right|<\epsilon$, and $\left|\Delta \mathcal{T}^{(i+1)} / \mathcal{T}^{(i)}\right|<\epsilon$ then convergence is achieved. The minimum period is computed based on Poincarè surface intersections that occur within the converged period $\mathcal{T}^{*}$.

\section{RESULTS AND DISCUSSION}

Unless mentioned otherwise, all effects appearing in the formulation are considered, and direct integration with the following data is used: Cutting parameters [3, 9, 10, 13]:Dia of work $=25 \mathrm{~mm}, t_{1_{e}}=0.15 \mathrm{~mm}, w=4.0 \mathrm{~mm}, V=200 \mathrm{~m} / \mathrm{min}, \alpha_{e}=38^{\circ}, m_{\mu}=$ $10^{-06}, \phi_{e}=50.6^{\circ}, \gamma_{e}=2^{0}, f_{s p}=4.1 \times 10^{5} \mathrm{~N} / \mathrm{mm}^{3}, \mu_{0}=\mu_{c}=0.3, \tau_{s}=700 \mathrm{~N} / \mathrm{mm}^{2}$, $\psi=0.0046 \mathrm{~mm}$; Vibration parameters [8]:- $m=40.87 \mathrm{~kg}, c_{x}=c_{y}=19.73 \mathrm{~N} . \mathrm{s} / \mathrm{mm}, \mu_{x}=$ $1.464, \mu_{y}=5.564$, and $k_{x}=k_{y}=180 \mathrm{kN} / \mathrm{m}$.

Figure 3(a) shows the transient $x$-chatter response for parameters which make the linearized system unstable. The displacement increases until a steady state (limit cycle) is slowly reached. The limit cycle trajectory (in the $\dot{x}^{-} x$ plane) obtained via both methods (i.e., direct integration and shooting) overlap. (Fig. 3(b)). The period, amplitude, and 

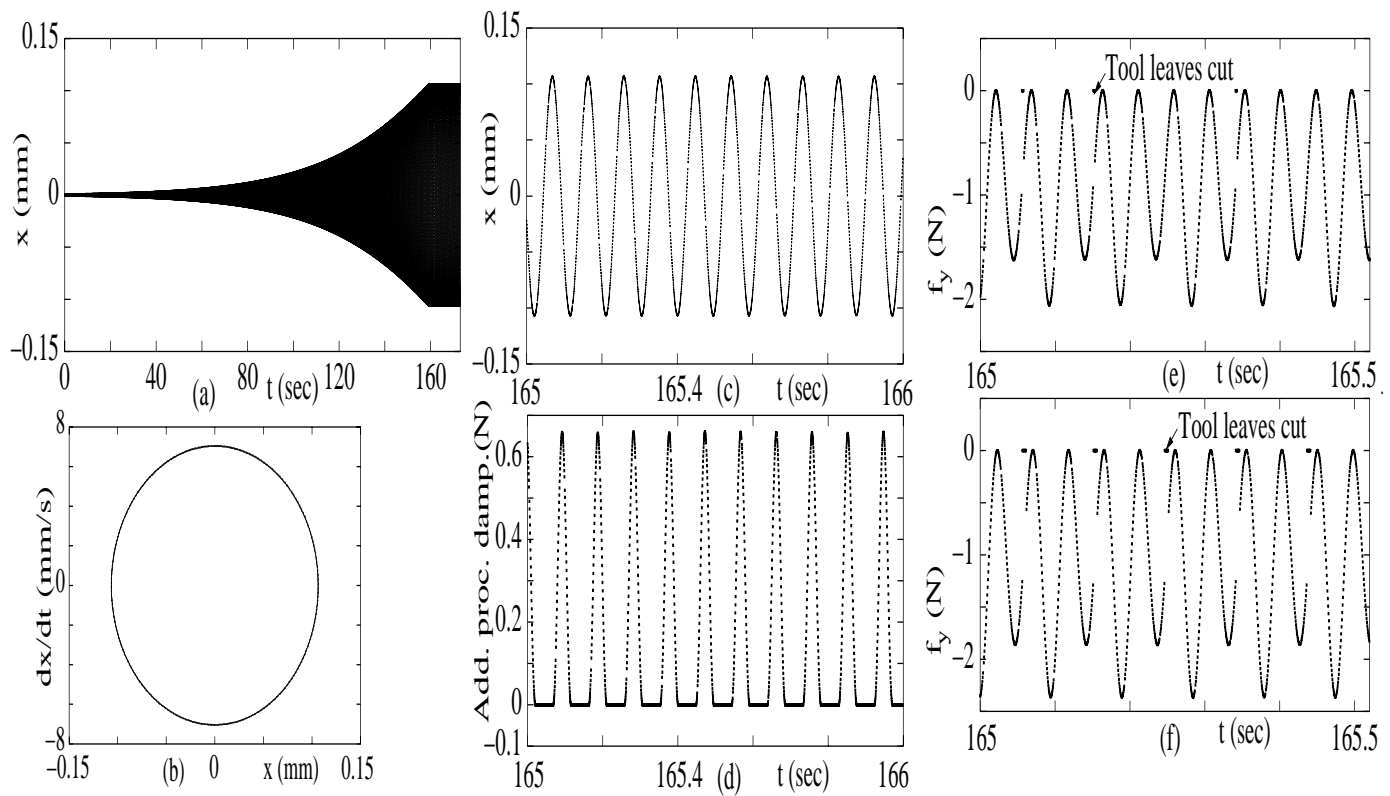

Figure 3: (a) Transient $x$-chatter; (b) Limit cycle; Steady state- (c) $x$-chatter, (d) Additional process damping force, (e,f) Ploughing force- $(\mathrm{e}) w=4 \mathrm{~mm},(\mathrm{f}) w=4.5 \mathrm{~mm}$

number of work revolutions for convergence are 0.00950141, 0.106324, and 7078, respectively, via direct integration, and 0.106292, 0.00950090, and 1492, respectively, via shooting. Steady state responses for $x$-chatter, additional damping force due to flank interference, and ploughing force are shown in Fig. 3(c, d, e, f). The additional damping vanishes when the tool moves upward (Fig. 3(c,d)). An increase in width-of-cut causes the ploughing force magnitude to increase and the tool to disengage from the work more frequently and for longer duration (Fig. 3(e,f)). Tool disengagement is characterized by the ploughing force suddenly vanishing.

Figure 4(a) shows the effect of the chatter-free uncut chip thickness on the limit cycle amplitudes obtained for varying widths of cut. At the critical width (i.e., $3.51 \mathrm{~mm}$ for $t_{1_{e}}=$ $0.5 \mathrm{~mm}$ and $3.64 \mathrm{~mm}$ for $t_{1_{e}}=0.15 \mathrm{~mm}$ and $0.3 \mathrm{~mm}$ ) the solution transforms from a fixed point to a limit cycle, i.e., a Hopf bifurcation occurs. For $t_{1_{e}}=0.15 \mathrm{~mm}$ and $0.3 \mathrm{~mm}$ a sudden jump to a finite-amplitude limit cyle occurs, thus indicating a subcritical bifurcation. Hence, for these cases finite amplitude instability (i.e., pre-chatter motion) is possible for sub-critical widths. As expected, the chatter amplitude increases with uncut chip thickness (since cutting forces increase with $t_{1_{e}}$ ). Figure $4(\mathrm{~b})$ shows amplitude versus width for cutting velocities $200 \mathrm{~m} / \mathrm{min}$ and $220 \mathrm{~m} / \mathrm{min}$. For a lower cutting speed, the jump occurs for a smaller width and the amplitude is higher. The jump phenomenon dissapears for larger $t_{1_{e}}$ (e.g., $0.5 \mathrm{~mm}$, results not displayed for brevity). An increase in additional process damping coefficients, $\mu_{x}$ and $\mu_{y}$, results in lower chatter amplitudes (for $t_{1_{e}}=0.5 \mathrm{~mm}$ ) as displayed in 

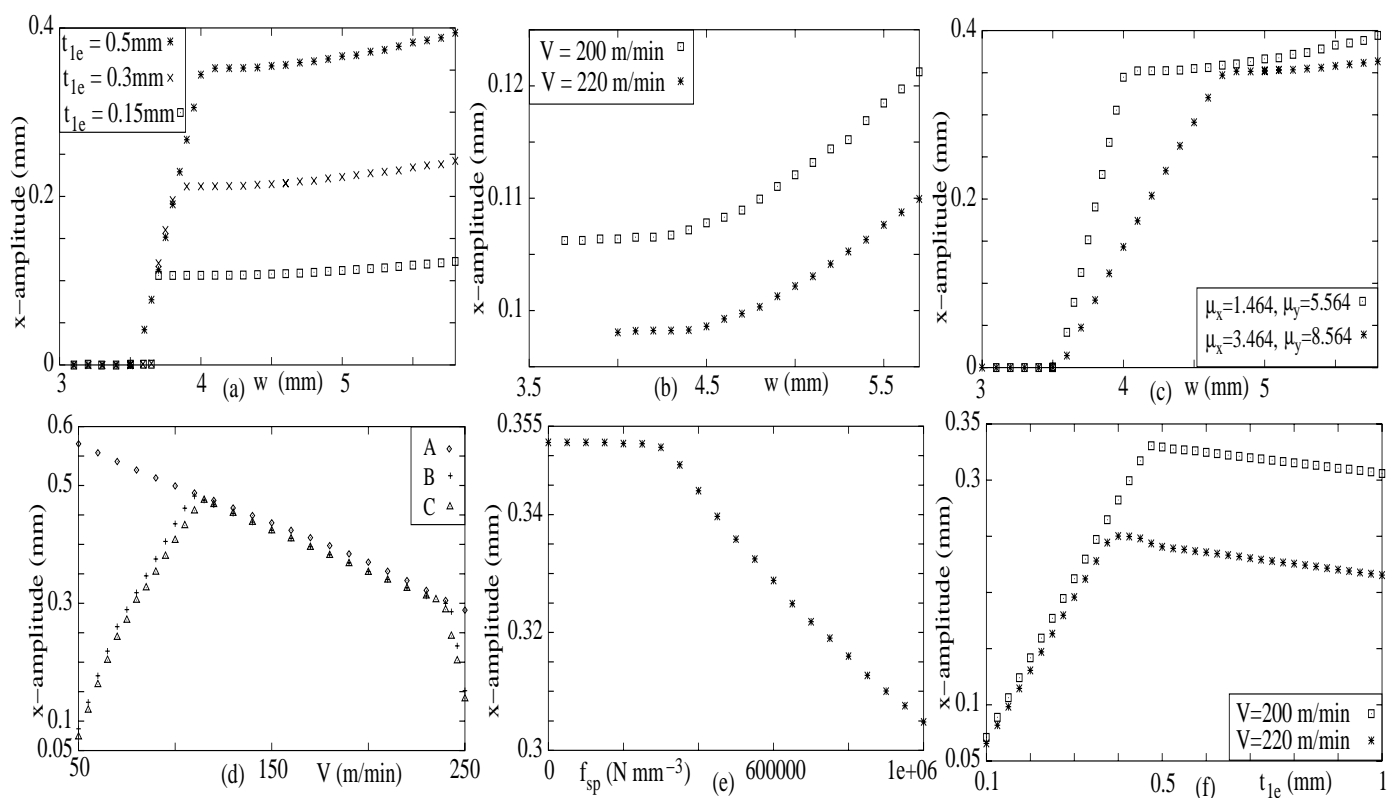

Figure 4: (a-c) Amplitude v/s Width-of-cut, effect of- (a) Uncut chip thickness, (b) Cutting speed, (c) Additional process damping coefficients $\left(t_{1_{e}}=0.5 \mathrm{~mm}\right)$; (d) Amplitude $\mathrm{v} / \mathrm{s}$ velocity ; (e) Amplitude v/s Ploughing force coefficient; (f) Amplitude v/s uncut chip thickness

Figure 4(c). A similar increase when $t_{1_{e}}=0.15 \mathrm{~mm}$ (result not displayed for brevity) causes the jump to occur at a larger width of cut. The effect of cutting velocity is shown in Fig. 4 (d) for various models. Model ' $C$ ' contains all effects, model ' $B$ ' neglects ploughing force and friction variation, and model ' $A$ ' further neglects flank intereference. Model $A$ predicts a decrease in amplitude when the cutting speed is increased. When additional process damping is included (model $B$ ), the amplitude at first increases with cutting speed and then (beyond $V=130 \mathrm{~m} / \mathrm{min}$ ) decreases. The amplitudes are lower for the additionally damped system (compare $A$ and $B$ ). A sudden increase in the rate of amplitude reduction occurs at $V=240 \mathrm{~m} / \mathrm{min}$ (model $B$ ). Further addition of ploughing force and friction variation effects (model $C$ ) yield a marginal reduction in amplitudes (compare $B$ and $C$ ). A fixed point (i.e., cessation of chatter) occurs beyond $V=250 \mathrm{~m} / \mathrm{min}$. Figure $4(\mathrm{e})$ confirms that while the effect of ploughing force is marginal for the value of $f_{s p}$ as considered here, it generally yields a reduction in amplitude. The variation in amplitude with the chatter-free uncut chip thickness is shown in Fig. 4(f). It is interesting to note that the trend of monotonically increasing amplitudes is reversed at higher chip thicknesses.

\section{CONLUSIONS AND SCOPE}

A comprehensive 2-DOF model of tool dynamics during turning has been considered 
and the following conclusions are made: (i) For the parameter ranges considered, period-1 motion describes the tool dynamics. (ii) Features of a subcritical Hopf bifurcation could appear in the amplitude versus width-of-cut plane, for certain parameter values. This implies the possibility of subcritical instability characterized by sudden onset of finite-amplitude chatter. (iii) Additional process damping causes a reduction in chatter amplitudes, and the subcritical instability to occur at a larger width of cut. (iv) An increase in width of cut causes frequent tool-leaving-cut events and increased chatter amplitudes. (v) The chatter amplitude at first increases and then decreases when the cutting velocity or the uncut chip thickness is increased. (vii) For the parameter ranges considered, variation in friction angle and/or ploughing force have a marginal effect. The shooting method could be made more efficient by using Newton Picard iterations. Structural nonlinearities could be included in the model. Hence, real-time active supression of chatter could be addressed.

\section{REFERENCES}

[1] Tobias, S. A., and Fishwick, W., The Chatter of Lathe Tools Under Orthogonal Cutting Conditions, Trans. ASME, 80, 1079-1088, 1958.

[2] Hanna, N. H. and Tobias, S. A., A Theory of Nonlinear Regenerative Chatter, Trans. ASME, J. Engng. Ind., 96, 247-255, 1974.

[3] Wu, D. W., and Liu, C. R., An Analytical Model of Cutting Tool Dynamics - Part 1: Model building, - Part 2: Verification, Trans. ASME, J. Engng. Ind., 107, 107-118, 1985.

[4] Berger, B. S., Rokni, M., and Minis, I., The Nonlinear Dynamics of Metal Cutting, Int. J. Engng. Sci., 30, 1433-1440, 1992.

[5] Johnson, M. A., and Moon, F. C., Nonlinear Techniques to Characterize Prechatter and Chatter Vibrations in the Machining of Metals, Int. J. Bif. \& Chaos, 11(2), 449-467, 2001.

[6] Tlusty, J., and Ismail, F., Basic Non-Linearity in Machining Chatter, Annals CIRP, 30(1), 299-304, 1981.

[7] Jemielniak, K., and Widota, A., Numerical Simulation of Non-linear Chatter Vibration in Turning, Int. J. Mach. Tools Manufact., 29(2), 239-247, 1989.

[8] Tarng, Y. S., Young, H. T., and Lee, B. Y., An Analytical Model of Chatter Vibration in Metal Cutting, Int. J. Mach. Tools Manufact., 34(2), 183-197, 1994.

[9] Wu, D. W., A New Approach of Formulating the Transfer Function for Dynamic Cutting Processes, Trans. ASME, J. Engng. Ind., 111, 37-47, 1989.

[10] Lee, B. Y., Tarng, Y. S., and Ma, S. C., Modeling of the Process Damping Force in Chatter Vibration, Int. J. Mach. Tools Manufact., 35(7), 951-962, 1995.

[11] Nosyreva, E. P., and Molinari, A., Analysis of Nonlinear Vibrations in Metal Cutting, Int. J. Mech. Sci., 40(8), 735-748, 1998.

[12] Stepan, G., Retarded Dynamical Systems: Stability and Characteristic Functions, Pitman Res. Notes in Mathematics (Vol. 210) John Wiley and Sons (New York), 1989.

[13] Lin, J. S., and Weng, C. I., Nonlinear Dynamics of Cutting Process, Int. J. Mech Sci., 33(8), 645-657, 1991.

[14] Luzyanina, T., Engelborghs, K., Lust, K., and Roose, D., Computation, Continuation and Bifurcation Analysis of Periodic Solutions of Delay Differential Equations, Int. J. Bifurcation and Chaos, 7(11), 2547-2560, 1997. 